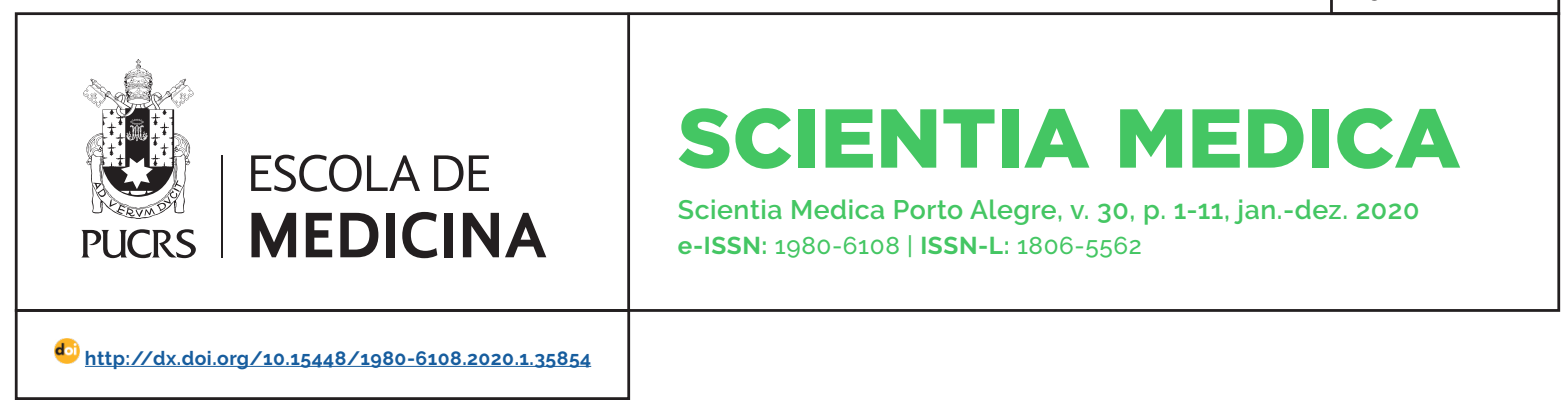

ARTIGO ORIGINAL

\title{
Conhecimento e atitudes de estudantes da área da saúde sobre a doação de córneas
}

\author{
Knowledge and attitudes toward corneal donation among health sciences \\ undergraduate students
}

\section{Isabela Mirandola Bartolomeu ${ }^{1}$ \\ orcid.org/0000-0002-5473-6414 \\ isabela.bartolomeu@usp.br}

\section{Sidney Júlio Faria e Sousa ${ }^{1}$}

orcid.org/0000-0003-3021-8476

sidneyjfs@gmail.com

\section{Miriane Lucindo \\ Zucoloto $^{1}$}

orcid.org/0000-0002-4745-227X

mirianezucoloto@gmail.com

\section{Edson Zangiacomi Martinez ${ }^{1}$}

orcid.org/0000-0002-0949-3222 edson@fmrp.usp.br

Recebido em: 1 out. 2019. Aprovado em: 12 jan. 2020. Publicado em: 14 abr. 2020.

\section{(c) (1)}

Artigo está licenciado sob forma de uma licença Creative Commons Atribuição 4.0 Internacional.

\section{Resumo}

Objetivos: investigar o conhecimento e as atitudes em relação ao transplante de córneas entre os acadêmicos de cursos de graduação da área da saúde.

Métodos: trata-se de um estudo observacional, descritivo, com delineamento transversal, que incluiu os alunos ativos dos cursos de graduação em Fonoaudiologia, Nutrição e Metabolismo, Fisioterapia ou Terapia Ocupacional, oferecidos pela Faculdade de Medicina de Ribeirão Preto (FMRP) da Universidade de São Paulo (USP), campus Ribeirão Preto. Os estudantes responderam em sala de aula a um instrumento estruturado. Foi aplicado um questionário contendo 16 itens sobre o conhecimento em relação ao transplante de córneas, baseado em instrumentos usados em trabalhos similares, e questões sobre as atitudes perante a doação.

Resultados: participaram da pesquisa 335 estudantes (83,1\% de sexo feminino). Apenas $6,6 \%$ declararam saber como proceder para ser um doador de córneas, e 55.5\% declararam saber que a retirada da córnea só pode ser realizada após o óbito do doador. Dentre os 16 itens do questionário de conhecimento, os estudantes tiveram uma média de apenas 5,6 respostas corretas, com um desvio padrão de 3,1 acertos. Conclusões: o conhecimento dos estudantes entrevistados sobre a doação de córnea é bastante insatisfatório. Diante da conhecida escassez de córneas para transplantes, são necessárias ações educativas que tornem esses futuros profissionais aptos a divulgarem a prática da doação de córneas entre seus futuros pacientes e respectivos familiares.

Palavras-chave: conhecimento, estudantes, transplante de córnea.

\section{Abstract}

Aims: assess the knowledge and attitudes toward corneal donation among health sciences undergraduate students.

Methods: this is an observational, descriptive, cross-sectional study, that included students enrolled in the undergraduate courses in Speech Therapy, Nutrition and Metabolism, Physical Therapy and Occupational Therapy, offered by the University of São Paulo at Ribeirão Preto Medical School (FMRP), University of São Paulo (USP), Ribeirão Preto campus. The students responded to a structured instrument in the classroom. A questionnaire containing 16 items regarding knowledge about corneal transplantation and questions about attitudes towards donation was applied. The questionnaire was based on instruments used in similar studies.

Results: 335 students (83.1\% female) participated in the research. Only 6.6\% reported knowing how to proceed to be a corneal donor, and 55.5\% reported knowing that corneal removal can only be performed after the donor's death. Among the 16 items of the knowledge questionnaire, the students had a mean of only 5.6 correct answers, with a standard deviation of 3.1.

Conclusions: the knowledge of the interviewed students about corneal donation is quite unsatisfactory. Given the known shortage of corneas for transplantation, educational actions are needed to make these future professionals able to spread the practice of corneal donation among their future patients and their families.

Keywords: knowledge, students, corneal transplantation. 
ABREVIATURAS: FMRP, Faculdade de Medicina de Ribeirão Preto; USP, Universidade de São Paulo.

\section{Introdução}

O transplante de córneas é o tipo mais comum de transplante de tecidos e constitui uma alternativa terapêutica cada vez mais eficiente para grande parte de doenças corneanas (1). A realização do transplante de córneas vem crescendo a cada ano no Brasil. Segundo a Associação Brasileira de Transplantes de Órgãos, em 2014 foram realizados 13.065 transplantes de córnea, aumentando para $14.531 \mathrm{em} 2016$ e se modificando discretamente nos anos seguintes, chegando a 14.809 transplantes no ano de 2018 (2). Os estados do Goiás, São Paulo e Ceará são os que possuem os maiores indices de transplante de córnea por milhão de população, sendo respectivamente 116,4, 113,8 e 104,0 no ano de 2018, enquanto o estado de São Paulo é o que mais se destaca em quantidade de pacientes que estão aguardando a cirurgia. No entanto, apesar de muitos transplantes realizados, esse número é ainda pequeno em relação à demanda necessária (3). Em 2018, por exemplo, a população do Brasil foi estimada em cerca de 208,5 milhões, sendo que, segundo a Associação Brasileira de Transplantes de Órgãos a demanda pelo transplante de córnea foi de 18.689, enquanto apenas 14.809 foram realizados. Na lista de espera, supõe-se que no Brasil em dezembro de 2018, havia 16.303 pacientes ativos à espera de um transplante de córnea, dos quais 539 eram pacientes pediátricos (2).

A disparidade existente entre o número de potenciais doadores e o número real de doações tem sido muito debatida na literatura cientíica (4-6). Acredita-se que entre os principais obstáculos para a doação de córneas nas populações esteja a falta de doadores e familiares esclarecidos, consequência da falta de conhecimento ou desinformação tanto por parte de profissionais da área da saúde quanto da população em geral, bem como entraves legais e religiosos $(4,7)$. Conforme um estudo norteamericano publicado em 1990 (8), dos 20 mil individuos que morrem por ano e que são doadores elegiveis, apenas 3 mil doam as córneas.
De acordo com Farge et al. (9), a presença de profissionais capacitados nos serviços de captação de órgãos e tecidos é indispensável para aumentar o número de doações. Em seu estudo, a maioria dos individuos que se autoclassificavam como não doador de córnea, tinham baixo conhecimento sobre o transplante, enquanto, entre os que tinham maior conhecimento, apenas $18,5 \%$ não eram doadores.

Em um estudo realizado por Rodrigues e Sato (4), foram entrevistadas pessoas que transitavam pelas enfermarias e entrada do pronto socorro de um hospital da cidade de São Paulo. Aproximadamente 95\% relataram que gostariam de obter mais informações sobre o transplante de córnea (4), evidenciando a necessidade de ampliar o conhecimento sobre o tema no ambiente hospitalar, tanto para a população de usuários quanto de profissionais da saúde.

Segundo Mack et al. (10), dois obstáculos mais importantes no processo de doação são a ausência de busca ativa por doadores e a entrevista familiar realizada de forma equivocada. A falta de uma busca ativa, segundo esses autores, está relacionada à educação escassa dos profissionais das áreas de saúde, inclusive os de terapia intensiva, já que essa é uma das principais fontes de doadores em potencial. Quanto à recusa familiar, a mesma poderia ser contornada mais facilmente se os profissionais envolvidos no processo de captação esclarecessem de forma competente as dúvidas dos familiares (10, 11).

A abordagem à família é um dos aspectos mais dificeis no processo de captação de órgãos. Muitos profissionais julgam-se incapazes e optam por não questionar a doação junto aos familiares, sendo essa a razão mais comum para a não efetivação da doação em potenciais doadores de acordo com alguns estudos (12-14). Além disso, considera-se que quando a abordagem é feita, o sucesso depende da experiência do profissional que a realizou (14).

Diante disso, é fundamental fomentar o interesse em doação de órgãos entre os estudantes, pois acredita-se que quanto mais precoce o contato com o tema, maior a possibilidade de esses buscarem informações e aprimoramento sobre o assunto (15). Os alunos, em breve, serão os 
profissionais que abordarão os familiares de potenciais doadores, por isso é importante obter esse conhecimento durante a graduação.

Assim, diante das evidências de que a falta de conhecimento tem um impacto negativo sobre a atitude da população frente à doação de córneas, a proposta deste estudo vem ao encontro da necessidade de investigar o conhecimento e as atitudes em relação ao transplante de córneas entre os acadêmicos dos cursos de graduação da Faculdade de Medicina de Ribeirão Preto (FMRP).

\section{Métodos}

Trata-se de um estudo descritivo observacional, sobre conhecimentos e atitudes. Quanto à temporalidade, trata-se de um estudo transversal, visto que os dados de cada participante foram obtidos em um único momento. Considerouse como critério de inclusão ser aluno ativo dos cursos de graduação em Fonoaudiologia, Nutrição e Metabolismo, Fisioterapia ou Terapia Ocupacional, oferecidos pela FMRP da Universidade de São Paulo (USP), campus Ribeirão Preto. Assim, a população inclui 565 estudantes matriculados nesses cursos. Esse número é baseado no número de alunos ingressantes em cada concurso vestibular e no número de anos necessários para o término do curso.

Os estudantes foram convidados a responder os instrumentos em sala de aula, ao término ou início da aula de alguma disciplina, com o consentimento do professor responsável e o compromisso de não prejudicar o andamento de sua aula. Em duplas, as entrevistadoras apresentaram a todos os estudantes presentes na sala de aula os objetivos da pesquisa e os convidaram a responder ao instrumento, perante a assinatura do Termo de Consentimento Livre e Esclarecido.

O presente estudo define o conhecimento como a compreensão a respeito de um tema específico. Marinho et al. (16) associam o conhecimento à habilidade que uma pessoa possui em aplicar fatos específicos para a resolução de problemas ou em emitir conceitos para o entendimento de um determinado evento. Assim, neste estudo, o conhecimento tem foco na posse de informações essenciais para atitudes perante a doação de córneas e a capacidade de motivar futuros pacientes e familiares à doação. Por sua vez, a atitude refere-se tanto aos sentimentos em relação a um tópico abordado, quanto às ideias preconcebidas que as pessoas podem ter a seu respeito (17). Segundo Marinho et al. (16), a atitude corresponde, essencialmente, a ter opiniões, mas também envolve sentimentos, predisposições e crenças, relativamente constantes.

Os participantes responderam a um questionário estruturado, abordando o sexo, a idade, o ano do curso de graduação, o tipo de residência, a religião e a avaliação do estado de saúde. Em adição, foi aplicado um questionário específico sobre o conhecimento em relação à doação de córnea, baseado em instrumentos usados em trabalhos similares $(6,18-22)$. 0 instrumento incluiu, ainda, a questão "Você sabe como proceder para ser um(a) doador(a) de córneas?", e outras questões relacionadas à atitude: "Você acredita que é uma pessoa apta a ser uma doadora da córneas?"; "Você apoiaria a decisão de um familiar ou amigo a ser um(a) doador(a) de córneas?"; e "Você estaria disposto a ser um(a) doador(a) de córneas?".

Os números médios de respostas corretas ao questionário de conhecimento foram comparados entre as classes de variáveis independentes de interesse utilizando modelos de regressão baseados na distribuição beta-binomial para dados de contagem (23). Esses modelos foram ajustados com o auxílio do pacote gamlss do programa R versão 3.4.3 (24), considerando um nivel de significância de 0,05.

O trabalho obteve a aprovação da Seção de Alunos da FMRP e do Comitê de Ética em Pesquisa do Hospital das Clínicas da FMRP, com parecer n. ${ }^{\circ}$ 2.667.586, conforme Resolução 466/2012 do Conselho Nacional de Saúde, que define normas para pesquisas envolvendo seres humanos.

\section{Resultados}

Dentre um total de 345 respondentes, dez estudantes foram excluidos por não preencher os itens do questionário de conhecimento. Assim, foi considerada para as análises uma casuística de 335 respondentes (59,3\% da população total), 


\section{4/11 Scientia Medica Porto Alegre, v. 30, p. 1-11, jan.-dez. 2020 | e-35854}

com idade entre 18 e 55 anos (média 20,9, desvio padrão 3,1 anos). A Tabela 1 mostra uma descrição da amostra, sendo que $83,1 \%$ dos respondentes são de sexo feminino. Nessa tabela, as frequências absolutas não somam 335 para algumas variáveis devido às ausências de respostas.

TABELA 1 - Descrição dos respondentes $(n=335)$

\section{Curso de graduação}

Fonoaudiologia

Nutrição e Metabolismo

Fisioterapia

Terapia Ocupacional

\section{Ano de ingresso na graduação}

2018

2017

2016

2015

2014 ou anterior

\section{Faixa etária (anos)}

$18-20$

$21-25$

Mais de 25 anos

Sexo

Feminino

Masculino

\section{Como reside}

Em uma república, moradia estudantil ou com outros estudantes

$152(45,5)$

Com os pais ou familia de origem

Sozinho(a)

Em uma pensão ou pensionato

Com outros familiares

$10(3,0)$

Com esposo(a), namorado(a) ou companheiro(a)
$275(83,1)$

$56(16,9)$

$88(26,3)$

$63(18,9)$

$14(4,2)$

$49(14,7)$

$23(6,9)$

$84(25,1)$

$38(41,2)$

$9(11,6)$

$89(28,7)$

$78(25,1)$

$52(16,7)$

$52(16,7)$

$(12,8)$

$164(50,2)$

$48(45.3)$

$5(4,5)$

$7(2,1)$

$23(6,9)$

\section{Possui religião}

Sim

Não, mas acredita em Deus

Não, ateu

Agnóstico(a) 


\section{Religião $(n=238)$}

$\begin{array}{lr}\text { Católica } & 142(59,7) \\ \text { Evangélica } & 43(18,1) \\ \text { Espírita } & 43(18,1) \\ \text { Outra } & 10(3,1)\end{array}$

\section{Avaliação do estado de saúde}

Boa

A Tabela 2 mostra que aproximadamente metade dos participantes acredita ser apta a tornar-se doadora de córneas (52,5), enquanto 44,5\% não sabe ou não respondeu. Uma frequência bastante grande de respondentes reportou que apoiaria um familiar ou amigo a tornar-se um doador de córneas (91,9\%). Doze respondentes declararam não estar dispostos a ser um doador de córneas. Dentre esses estudantes, oito justificaram sua resposta afirmando que necessitam mais informações sobre como é o procedimento, cinco declararam que nunca pensaram nessa possibilidade, e dois declararam que suas famílias não apoiariam essa decisão. Outras justificativas incluiram a crença que o rosto sofreria deformações, a possibilidade de ser vítima de tráfico de órgãos e o medo do procedimento.

TABELA 2 - Descrição das atitudes dos respondentes $(n=335)$

\begin{tabular}{|c|c|c|c|}
\hline \multirow{2}{*}{ Questão } & Sim & Não & $\begin{array}{l}\text { Não sabe ou não } \\
\text { respondeu }\end{array}$ \\
\hline & $n(\%)$ & $n(\%)$ & $n(\%)$ \\
\hline $\begin{array}{l}\text { Você acredita que é uma pessoa apta a tornar-se uma } \\
\text { doadora da córneas? }\end{array}$ & $176(52,5)$ & $10(3,0)$ & $149(44,5)$ \\
\hline $\begin{array}{l}\text { Você apoiaria a decisão de um familiar ou amigo a ser } \\
\text { um(a) doador(a) de córneas? }\end{array}$ & $308(91,9)$ & $1(0,3)$ & $26(7,8)$ \\
\hline Você estaria disposto a ser um(a) doador(a) de córneas? & $225(67,2)$ & $12(3,6)$ & $98(29,2)$ \\
\hline
\end{tabular}

Apenas 22 (6,6\%) participantes declararam saber como proceder para ser um doador de córneas. Em adição, a Tabela 3 mostra que em todos os itens do questionário de conhecimento sobre doação de córneas observam-se frequências relativamente grandes de estudantes que não responderam ou declararam não saber a resposta. Observa-se que apenas 55,5\% dos estudantes sabem que a doação de córnea só pode ser realizada após o óbito do doador, e um baixo conhecimento em relação às condições de saúde que impedem a doação (apenas 28,7\% sabem que portadores do vírus HIV não podem doar, enquanto 19,4\% sabem que portadores de hepatite B ou C também não podem tornar-se doadores). 
TABELA 3 - Frequências das respostas ao questionário de conhecimento sobre doação de córneas $(n=335)$

\begin{tabular}{|c|c|c|c|}
\hline \multirow{2}{*}{ Questão } & Verdadeiro & Falso & $\begin{array}{l}\text { Não sabe ou } \\
\text { não respondeu }\end{array}$ \\
\hline & $n(\%)$ & $n(\%)$ & $n(\%)$ \\
\hline $\begin{array}{l}\text { Nos transplantes de córnea, os familiares do doador são con- } \\
\text { sultados e precisam autorizar a doação }\end{array}$ & $210(62,7)^{*}$ & $20(6,0)$ & $105(31,3)$ \\
\hline $\begin{array}{l}\text { A retirada do tecido para a doação pode modificar a aparência } \\
\text { do doador ou causar deformações }\end{array}$ & $21(6,3)$ & $151(45,1)^{*}$ & $163(48,7)$ \\
\hline $\begin{array}{l}\text { Pessoas com problemas da visão como miopia, hipermetro- } \\
\text { pia, astigmatismo, catarata e glaucoma podem ser doadoras } \\
\text { de córnea }\end{array}$ & $60(17,9)^{*}$ & $87(26,0)$ & $188(56,1)$ \\
\hline A retirada da córnea só pode ser feita após a morte do doador & $186(55.5)^{*}$ & $25(7,5)$ & $124(37,0)$ \\
\hline $\begin{array}{l}\text { Pessoas portadoras do vírus HIV podem ser doadoras de } \\
\text { córnea }\end{array}$ & $14(4,2)$ & $96(28,7)^{*}$ & $225(67,2)$ \\
\hline $\begin{array}{l}\text { Pessoas portadoras de diabetes ou hipertensão podem ser } \\
\text { doadoras de córneas }\end{array}$ & $67(20,0)^{*}$ & $38(11,3)$ & $230(68,7)$ \\
\hline No Brasil, existe um cadastro nacional de doadores de córneas & $198(59,1)$ & $3(0,9)^{*}$ & $134(40,0)$ \\
\hline $\begin{array}{l}\text { Pessoas portadoras de hepatite B ou C podem ser doadoras } \\
\text { de córneas }\end{array}$ & $6(1,8)$ & $65(19,4)^{*}$ & $264(78,8)$ \\
\hline $\begin{array}{l}\text { O familiar de um doador falecido pode escolher quem rece- } \\
\text { berá a córnea }\end{array}$ & $27(8,1)$ & $159(47.5)^{*}$ & $149(44,5)$ \\
\hline $\begin{array}{l}\text { A retirada das córneas só ocorrerá depois de comprovada a } \\
\text { morte encefálica do doador }\end{array}$ & $173(51,6)^{*}$ & $13(3,9)$ & $149(44,5)$ \\
\hline No procedimento de doação, retira-se todo o globo ocular & $25(7,5)^{*}$ & $70(20,9)$ & $240(71,6)$ \\
\hline $\begin{array}{l}\text { A causa da morte de um doador deve ser necessariamente } \\
\text { conhecida }\end{array}$ & $175(52,2)^{*}$ & $21(6,3)$ & $139(41,5)$ \\
\hline $\begin{array}{l}\text { Em um transplante de córnea, deve haver compatibilidade de } \\
\text { grupos sanguineos entre doador e transplantado }\end{array}$ & $114(34,0)$ & $30(9,0)^{*}$ & $191(57,0)$ \\
\hline $\begin{array}{l}\text { Nos transplantes de córnea, é possivel haver rejeição após o } \\
\text { procedimento }\end{array}$ & $227(67,8)^{*}$ & $5(1,5)$ & $103(30,7)$ \\
\hline $\begin{array}{l}\text { Nos transplantes de córnea, o transplantado passa a ter a cor } \\
\text { dos olhos do doador }\end{array}$ & $19(5,7)$ & $155(46,3)^{*}$ & $161(48,1)$ \\
\hline $\begin{array}{l}\text { Considerando um doador falecido, o procedimento de doa- } \\
\text { ção pode causar atrasos no velório ou funeral }\end{array}$ & $31(9,3)$ & $126(37,6)^{*}$ & $178(53,1)$ \\
\hline
\end{tabular}

* indica a resposta correta.

Dentre os 16 itens que compõem o questionário de conhecimento, os participantes tiveram uma média de apenas 5,6 respostas corretas, com um desvio padrão de 3,1 acertos. A Tabela 4 exibe as médias e os desvios padrão dos números de respostas corretas do questionário de conhecimento sobre doação de córneas e valores $p$ obtidos do ajuste do modelo beta-binomial de regressão. Os valores $p$ associados às variáveis curso de graduação, ano de ingresso na graduação e sexo foram obtidos do ajuste de um único modelo múltiplo, de forma que possiveis efeitos de confundimento entre essas variáveis fossem minimizados. Os valores $p$ associados às faixas etárias foram obtidos de 
outro ajuste do modelo de regressão, inserindo o curso de graduação e o sexo como possiveis variáveis de confundimento. Não foi possível ajustar um único modelo de regressão incluindo todas essas variáveis conjuntamente, dado um forte efeito de colinearidade observado entre o ano de ingresso e as faixas etárias. Assim, observa- se um menor conhecimento sobre doação de córneas entre estudantes do curso de graduação em Nutrição e Metabolismo, entre os ingressantes no ano da coleta dos dados (2018), e entre os mais jovens (idade de 18 a 20 anos, respectivos valores p menores que 0,05).

TABELA 4 - Médias e desvios padrão dos números de respostas corretas questionário de conhecimento sobre doação de córneas $(n=335)$.

\begin{tabular}{|c|c|c|c|c|}
\hline Variável & $n$ & Média & Desvio padrão & Valor $p$ \\
\hline \multicolumn{5}{|l|}{ Curso de graduação } \\
\hline Fonoaudiologia & 84 & 6,21 & 2,94 & Referência \\
\hline Nutrição e Metabolismo & 74 & 5,09 & 3.36 & 0,02 \\
\hline Fisioterapia & 138 & 5,62 & 3,05 & 0,20 \\
\hline Terapia Ocupacional & 39 & 5.97 & 3,21 & 0,63 \\
\hline \multicolumn{5}{|c|}{ Ano de ingresso na graduação } \\
\hline 2018 & 89 & 4,82 & 2,96 & Referência \\
\hline 2017 & 78 & 5,82 & 2,87 & 0,03 \\
\hline 2016 & 52 & 6,04 & 2,93 & 0,02 \\
\hline 2015 & 52 & 6,06 & 3.59 & 0,04 \\
\hline 2014 ou anterior & 40 & 6,70 & 3,08 & $<0,01$ \\
\hline \multicolumn{5}{|l|}{ Faixa etária (anos) } \\
\hline $18-20$ & 164 & 5,13 & 3,01 & Referência \\
\hline $21-25$ & 148 & 6,32 & 3,14 & $<0,01$ \\
\hline Mais de 25 anos & 15 & 5.93 & 3,61 & 0,57 \\
\hline \multicolumn{5}{|l|}{ Sexo } \\
\hline Feminino & 275 & 5,89 & 3,01 & Referência \\
\hline Masculino & 56 & 4,61 & 3,49 & 0,06 \\
\hline
\end{tabular}

Os valores $p$ foram obtidos do ajuste do modelo múltiplo beta-binomial de regressão.

\section{Discussão}

Dentre 335 participantes, apenas $22(6,6 \%)$ declararam saber como proceder para ser um doador de córneas. Em adição, a Tabela 3 descreve uma alta frequência de estudantes que não responderam ou declararam não saber as respostas aos itens do questionário de conhecimento, e apenas 55.5\% desses estudantes declararam saber que a retirada da córnea só pode ser realizada após o óbito do doador. No Brasil, o transplante de órgãos somente pode ser feito após a morte encefálica do doador, após o obrigatório consentimento familiar $(25,26)$. Em um estudo que incluiu estudantes nigerianos do último ano de um curso de Medicina, $72,5 \%$ não sabiam que as córneas necessitam ser retiradas de um doador falecido (27). De forma geral, muitos estudos considerando estudantes do Brasil $(18,21)$ e de outros países (28-39) têm mostrado muitas deficiências no conhecimento sobre a doação de córneas. 
Quando indagados se no procedimento da doação, retira-se todo o globo ocular (enucleação), 7.5\% acreditam que sim, e 20,9\% acreditam que não (Tabela 3). Embora a opção de se remover somente as córneas seja viável e comum em alguns paises (35), a enucleação do globo ocular é considerada mais fácil, possibilitando uma melhor avaliação do tecido doado e permitindo que a esclera seja também armazenada (40). Em adição, estima-se que a superficie de $80 \%$ dos olhos enucleados é colonizada por bactérias, o que leva a riscos de transferências de germes e consequente infecção intraocular em transplantes $(41,42)$. Considerando que o descarte de córneas com contaminação da superfície ocular é inviável, dado que as perdas seriam graves perante a demanda de córneas para transplantes, as medidas tomadas para a redução destes riscos incluem banho de imersão do globo ocular em colírio de ciprofloxacino e uso de soluções de preservação de tecido que contém antibióticos (42). Entretanto, essas medidas exigem a enucleação, o que nos levou a considerar a resposta "verdadeira" como a mais adequada ao item "no procedimento de doação, retira-se todo o globo ocular" do instrumento de conhecimento. Observa-se que esse item foi aquele com menos repostas na presente pesquisa (78,8\% declararam não saber ou não responderam). Embora esse conhecimento seja bastante técnico, pode ser útil ao profissional da saúde em atividades de educação da população, considerando que a ideia da retirada completa de órgãos pode ser um potencial obstáculo à decisão de tornar-se um doador ou de permitir a retirada de órgãos de parentes. No caso da doação de córneas, isso inclui crenças religiosas e místicas, como o medo do doador sentir dor na remoção do órgão e a necessidade dos olhos no além-vida (22,

27. 43). Sousa e Barretto (43) discutem como essas objeções relativas à doação são trabalhadas nas entrevistas da familia para a obtenção de órgãos e tecidos para transplantes, sendo importantes recursos ao profissional da saúde.

Foi observado que 45,1\% dos entrevistados acredita ser falsa a afirmativa que a doação pode modificar a aparência do doador ou causar deformações. A Lei n. ${ }^{0} 10.211$, de 23 de março de 2001, que altera dispositivos da Lei n. ${ }^{\circ}$ 9.434, de 4 de fevereiro de 1997 (26), dispõe que "Após a retirada de tecidos, órgãos e partes, o cadáver será imediatamente necropsiado, se verificada a hipótese do parágrafo único do art. $7^{\circ}$, e, em qualquer caso, condignamente recomposto para ser entregue, em seguida, aos parentes do morto ou seus responsáveis legais para sepultamento". Por outro lado, em alguns casos, a retirada do globo ocular pode causar deformações na vigência de hemorragia profusa na órbita. $\mathrm{Na}$ interpretação das respostas ao instrumento de conhecimento, consideramos "falsa" a resposta correta para o item "A retirada do tecido para a doação pode modificar a aparência do doador ou causar deformações", dado que as deformações não ocorrem na maioria dos casos. Entretanto, recomendamos a revisão desse item em aplicações futuras do instrumento.

De modo geral, observa-se que os participantes tiveram uma tendência a, em maior frequência, responder corretamente aos itens do questionário de conhecimento quando esses envolviam questões também embasadas em legislação específica, e não somente a aspectos fisiológicos ou patológicos. Observa-se que 62,7\% dos estudantes sabem que nos transplantes de córnea, os familiares do doador devem ser consultados e precisam autorizar a doação, o que é sustentado no Capítulo II, Artigo $4{ }^{\circ}$, da Lei n. ${ }^{\circ}$ 9.434: "A retirada de tecidos, órgãos e partes do corpo de pessoas falecidas para transplantes ou outra finalidade terapêutica, dependerá da autorização do cônjuge ou parente [...]" (26). Em adição, 55.5\% sabe que a doação só pode ser feita após a morte do doador, 51,6\% acredita que a retirada das córneas só poderá ocorrer após a comprovação da morte encefálica do doador, e 52,2\% conhecem que a causa da morte do doador deve ser necessariamente conhecida. Esses preceitos também estão presentes no Capítulo II da Lei n. 9.434 (26). Por outro lado, tratando-se de futuros profissionais da saúde, um percentual relativamente baixo de respondentes 
sabe que pessoas portadoras de hepatite B e C $(19,4 \%)$ ou do virus HIV $(28,7 \%)$ não podem ser doadoras de córneas. Em adição, 34,0\% acreditam que em transplantes de córnea, deve haver compatibilidade de grupos sanguíneos entre doador e transplantado. Dezenove estudantes $(5.7 \%)$ acreditam que nos transplantes, o transplantado passa a ter a cor dos olhos do doador, e 48,1\% não soube responder a esse item. Isso reforça um conhecimento insatisfatório, evidenciando que muitos alunos da área da saúde desconhecem que a cor dos olhos é definida pela íris, situada na parte anterior do cristalino e posterior da córnea, e não pela córnea.

Entre as limitações do estudo, usamos uma amostra de estudantes de graduação de um campus de uma única faculdade brasileira, o que dificulta a extrapolação dos resultados para amostras maiores. Em adição, os quatro cursos de graduação escolhidos para a composição da amostra não são representativos de todos os cursos da área da saúde. Da população total de 565 estudantes matriculados, 40,7\% não participaram da pesquisa, considerando as recusas e a possiblidade de não estarem presentes na sala de aulas durante a coleta de dados. Em relação ao instrumento utilizado, recomenda-se algumas revisões para estudos futuros, como uma avaliação por especialistas quanto à abrangência dos itens e aperfeiçoamentos de questões como a referente às possiveis modificações da aparência do doador ou deformações, conforme discutido anteriormente.

Apesar dessas limitações, os achados do estudo permitem constatar que o conhecimento dos estudantes entrevistados sobre doação de córnea é bastante insatisfatório. Esse resultado é compatível com o encontrado por outros autores em estudos com populações de estudantes brasileiros e de outros países $(18,21$, 27-39). Portanto, diante da escassez de córneas para transplantes descrita pela literatura, são necessárias ações educativas que tornem esses futuros profissionais aptos a divulgar a prática da doação de córneas entre seus futuros pacientes e respectivos familiares.

\section{Referências}

1. Farge EJ, Sulverman ML, Khan MM, Wilhelmus KR. The impact of state legislation on eye banking. Arch Ophtalmol. 1994;112(2):180-5.

2. Associação Brasileira de Transplantes de Órgãos. Registro Brasileiro de Transplantes. Dimensionamento de transplantes no Brasil e em cada estado (2011-2018) [periódico na internet]. 2018. Disponível em: http://ww/w.abto.org.br/abtovo3/Upload/file/RBT/2018/LV_RBT-2018.pdf. https://doi org/10.15329/2318-0498.20150009

3. Almeida HG, Hida RY, Kara-Junior N. Trends in corneal transplantation from 2001 to 2016 in Brazil. Arq Bras Oftalmol. 2018;81(6):529-38.

4. Rodrigues AM, Sato EH. Knowledge and attitude of the general population regarding corneal transplantation. Arq Bras Oftalmol. 2002;65(6):637-40.

5. Loewnetein A, Rahmiel R, Varssano D, Lazar IM. Obtaining consent for eye donation. Isr J Med Sci. 1991;27(2):79-81.

6. Rodrigues AM, Sato EH. The knowledge of the intensive care physicians on corneal donation. Arq Bras Oftalmol. 2003;66(1):29-32.

7. Ishhay R. Eye donation - how to maximize procurement. Isr J Med Sci. 1991; 27:89-91.

8. McGough EA, Chopek MW. The physician's role as asker in obtaining organ donations. Transplant Proc. 1990;22(1):267-72.

9. Farge EJ, Silverman ML, Khan MM, Wilhelmus KR. The impact of state legislation on eye banking Arch Ophthalmol. 1994:112(2):180-5. https://doi. org/10.1001/archopht.1994.01090140056023.

10. Mack JR, Mason P. Matheus WD. Obstacles to donor eye procurement and their solutions at the University of lowa. Cornea. 1995:14(3):249-52. https:// doi.org/10.1097/00003226-199505000-00004

11. Muraine M, Menguy E, Martin J, Sabatier P, Watt L, Brasseur $\mathrm{G}$. The interview with the donor's Family before post mortem procurement. Cornea. 2000;19(1):12-6.

12. Amaral ASR, Roza BA, Galvão FHF, Jardim KM, Medicina-Pestana JO. Knowledge of organ donation among one group of Brazilian professor of medicine. Transplant Proc. 2002:34(2):449-50. https://doi. org/10.1016/s0041-1345(02)02591-5

13. Tandon R, Verma K, Vanathi M, Pandey RM, Vajpayee R. Factors affecting eye donation from potmortem cases in a tertiary care hospital. Cornea. 2004; 23(6):597-601. https://doi.org/10.1097/01. ico.0000121706.58571.f6 
14. Murraine M, Menguy E, Martin J, Sebatier P. Watt $L$, Brasseur $G$. The interview with the donor's family before postmortem Cornea procurement. Cornea. 2000; 19(1):12-6. https://doi.org/10.1097/00003226200001000-00003

15. Bardell T, Hunter Dj, Kent WD, Jain MK. Do medical students have the knowledge needed to maximixe organ donation rates? Can J Surg. 2003; 46(6): 453-7.

16. Marinho LAB, Costa-Gurgel MS, Cecatti JG, Osis MJD. Conhecimento, atitude e prática do auto-exame das mamas em centros de saúde. Rev Saúde Pública. 2003; 37(5): 576-82. https://doi.org/10.1590/ s0034-89102003000500005

17. Kaliyaperumal K. Guideline for conducting a knowledge, attitude and practice (KAP) study. AECS Illumination. 2004; 4(1):7-9

18. Espidola RF, Rodrigues BA, Penteado LT, Tan-Ho G, Gozzan JOA, Freitas JAH. Conhecimento de estudantes de medicina sobre o processo de doação de córneas. Arq Bras Oftalmol. 2007:70(4):581-84. https://doi.org/10.1590/s0004-27492007000400004

19. Krieger MAL, Novellino MMM, Mariushi ACM, Moreira $\mathrm{H}$. Avaliação do conhecimento da população e dos profissionais da saúde sobre doação de córneas. J Bras Transpl. 2009; 12:1054-8.

20. Rosa LPD, Ventura L, Duarte SKM, Adam Netto A. Evaluation of knowledge of physicians with specialty in medical clinic and surgical clinic about the process of corneas donation. Rev Bras Oftalmol. 2017; 76(2):7480. https://doi.org/10.5935/0034-7280.20170015

21. Ramos APP. Avaliação do conhecimento dos estudantes do curso de graduação em medicina na Universidade Federal de Santa Catarina sobre o processo de doação de córneas para transplante. [Trabalho de Conclusão de Curso]. [FlorianópoLis]: Universidade Federal de Santa Catarina; 2009. 38 p. Disponivel em: https://repositorio.ufsc.br/ xmlui/handle/123456789/120299. https://doi. org/10.20873/uft.2525-4863.2017v2n3p965

22. Lawlor M, Kerridge I, Ankeny R, Dobbins TA, Billson F. Specific unwillingness to donate eyes: the impact of disfigurement, knowledge and procurement on corneal donation. Am J Transplant. 2010; 10(3):657-63. https://doi.org/10.1111/j. 1600-6143.2009.02986.x

23. Griffiths DA. Maximum likelihood estimation for the beta-binomial distribution and an application to the household distribution of the total number of cases of a disease. Biometrics. 1973; 29(4):637-48. https://doi.org/10.2307/2529131

24. Stasinopoulos DM, Rigby RA. Generalized additive models for location scale and shape (GAMLSS) in R. J Stat Softw. 2007; 23(7):1-46. https://doi. org/10.18637/jss.v023.io7
25. Marinho A, Cardoso SDS, Almeida VVD. Organ transplantation in Brazilian States: effectiveness, productivity, and capacity. Cad Saúde Pública. 2011; 27(8): 1560-8.

26. Brasil. Lei $n^{\circ}$ 9.434, de 4 de Fevereiro de 1997 Dispõe sobre a remoção de órgãos, tecidos e partes do corpo humano para fins de transplante e tratamento e dá outras providências. Icitado 2014 Out 12]. Disponivel em: http://Www.planalto.gov.br/ccivil_03/leis/l9434.htm. https://doi.org/10.1590/0034716719730005000016

27. Okoye O, Maduka-Okafor FC, Eze BI. What does the medical student know about eye donation/corneal transplant? The University of Nigeria scenario. West Indian Med J. 2010; 59(1): 41-4.

28. Singh MM, Rahi M, Pagare D, Ingle GK. Medical students' perception on eye donation in Delhi. Indian J Ophthalmol. 2007; 55(1): 49. https://doi. org/10.4103/0301-4738.29495

29. Kumar S, Shukla US, Agarwal P. Awareness and knowledge on eye donation among students at Bhopal. Natl J Community Med. 2012; 3(4): 685-9.

30. Nekar MS, Lokare L, Gokhale SA, Godbole M, Mulkipatil SY, MaHesh V. Awareness of eye donation among college students of Hubli city, Karnataka. Int J Biomed Res. 2012; 3(4):201-4. https://doi. org/10.7439/ijbr.v3i4.415

31. Eze BI, Okoye O, Eze JN. Knowledge and attitudes regarding eye donation and corneal transplant: medical versus nonmedical university students in a developing country in Africa. Exp Clin Transplant. 2014; 12(5): 454-61.

32. Sadana A, Sushma M, Lekha KC, Dudala SR, Ravi G, Prabhu K. Assessment of knowledge and attitude regarding eye donation among undergraduate medical students, Tirupati. Int J Med Pharm Sci. 2014; 4(9):9-24

33. Hameed N, Jadidy E. Knowledge and attitude regarding eye donation and corneal transplantation among medical students of Taibah University in Medina, Saudi Arabia 2015. Int J Acad Sci Res. 2015; 3(4):18-26.

34. Vidusha K, Manjunatha, S. Awareness of eye donation among medical students of tertiary care hospital, Bangalore. Asian Pac J Health Sci. 2015; 2(2): 94-8. https://doi.org/10.21276/apjhs.2015.2.2.15

35. Paraz CMA, Truong HTT, Sai DK, Cajucom-Uy HY, Chan CLL, Kassim SM. Knowledge and attitudes toward corneal donation among Singaporean youth: a cross-sectional study. Eye and Vision. 2016; 3(1): 17. https://doi.org/10.1186/s40662-016-0049-3

36. Gupta PC, Duggal M, Jamir L, Sharma D, Kankaria A, Sathyanath S, Kaur R, Rana K, Ram J. Knowledge and attitude toward corneal donation among high school children in northern India. Cornea. 2017; 36(5):611-6. 
37. Acharya M, Chaku D, Dave A, Das A, Patel NV. Knowledge and attitude toward corneal donation among high school children in northern India. Cornea. 2018; 37(1): e2-e3. https://doi.org/10.1097/ ic0.0000000000001372

38. Al-Labadi L, Gammoh Y, Shehada R, Shahin R, Jbarah W, Amro M, Athamny H. University students knowledge of corneal donation and willingness to donate corneas in the occupied Palestinian territory: a cross-sectional study. Lancet. 2018; 391: S22. https://doi.org/10.1016/s0140-6736(18)30388-x

39. Ahmad S, Ali M, Afzal M. Knowledge and attitudes toward corneal donation amongst medical students in University of Lahore (a cross-sectional study). Ophthalmol Update. 2018; 16(1):465:581.

40. Garcia VD, Abud Filho M, Neumann J. Transplante de órgãos e tecidos. $2^{\circ}$ ed. São Paulo: Segmento Farma, 2006

41. Panda A, Saxena R, Vajpayee RB, Satpathy G, Angra SK, Sethi HS. The efficacy of postenucleation saline wash and theeffect of different antimicrobial agents on microbial contamination of donor eyes. Ophthalmic Res. 2006;38(5):287-93.

42. Marcomini LAG, Sobral RMGR, Seixas GO, Sousa SJDF. Seleção de córneas para transplantes. Rev Bras Oftalmol. 2011; 70(6): 430-6.

43. Sousa SJDF, Barretto S. Family interview for the procurement of organs and tissues for transplantation. Arq Bras Oftalmol. 1999; 62(6):759-62.

\section{Isabela Mirandola Bartolomeu}

Bacharel em Fisioterapia pela Faculdade de Medicina de Ribeirão Preto, Universidade de São Paulo (FMRP-USP). Ribeirão Preto, SP, Brasil.

\section{Sidney Júlio Faria e Sousa}

Doutor em Oftalmologia pela Faculdade de Medicina de Ribeirão Preto, Universidade de São Paulo (FMRP-USP), professor da Universidade de São Paulo (USP). Ribeirão Preto, SP, Brasil.

\section{Miriane Lucindo Zucoloto}

Doutora em Ciências da Saúde pela Faculdade de Medicina de Ribeirão Preto, Universidade de São Paulo (FMRP-USP), pós-doutoranda da Universidade de São Paulo (USP). Ribeirão Preto, SP, Brasil.

\section{Edson Zangiacomi Martinez}

Doutor em Ciências da Saúde pela Universidade Estadual de Campinas (UNICAMP), professor da Universidade de São Paulo (USP). Ribeirão Preto, SP, Brasil.

\section{Endereço para correspondência}

Edson Zangiacomi Martinez

Faculdade de Medicina de Ribeirão Preto da Universidade de São Paulo (FMRP-USP)

Av. Bandeirantes, 3900

Monte Alegre, 14049-900

Ribeirão Preto, SP, Brasil

Notas

\section{Apoio financeiro}

Este estudo recebeu apoio financeiro da PróReitoria de Graduação da Universidade de São Paulo (USP)

\section{Declaração de conflito de interesses}

Os autores declaram não haver conflitos de interesses relevantes ao conteúdo deste estudo.

\section{Contribuições dos autores}

Todos os autores fizeram contribuições substanciais para concepção, ou delineamento, ou aquisição, ou análise ou interpretação de dados; e redação do trabalho ou revisão crítica; e aprovação final da versão para publicação. 